\title{
Effects of Emotional Stability on Insomnia through Anxiety in Patients with Cataracts
}

\author{
In-Young Ahn', So-Jin Lee ${ }^{2,3}$, Yong-Seop Han ${ }^{4}$, Chul-Soo Park ${ }^{2,3}$, Bong-Jo Kim, \\ Cheol-Soon Lee ${ }^{1,3}$, Boseok Cha ${ }^{2,3}$, Ji-Yeong Seo', Jae-Won $\mathrm{Choi}^{2}$, and Dongyun Lee ${ }^{1 凶}$ \\ 1'Department of Psychiatry, Gyeongsang National University Changwon Hospital, Changwon, Republic of Korea \\ ${ }^{2}$ Department of Psychiatry, Gyeongsang National University Hospital, Jinju, Republic of Korea \\ ${ }^{3}$ Department of Psychiatry, Gyeongsang National University College of Medicine, Jinju, Republic of Korea \\ ${ }^{4}$ Department of Ophthalmology, Gyeongsang National University Changwon Hospital, Changwon, Republic of Korea
}

This study investigated the effect of emotional stability (ES) on insomnia through anxiety in preoperative patients with cataracts. Fiftyfour subjects completed a self-questionnaire that included the Insomnia Severity Index (ISI), the International Personality Item Pool (IPIP) for ES, and the Hospital Anxiety and Depression scale (HADS). Pathway analysis was performed to analyze the mediating effects of ES, the anxiety subscale scores of the HADS, and the ISI scores. The low-ES group exhibited an insomnia severity that was significantly higher than that of the high-ES group ( $\mathrm{p}=0.048$ ). According to the pathway analysis, the significant indirect effect of ES on the ISI scores was mediated by the scores on the anxiety subscale of the HADS. Cataract patients with low ES waiting to undergo surgery have a high risk of experiencing insomnia due to anxiety. Before surgery, it is essential to identify patients' personality characteristics and provide appropriate intervention.

Psychiatry Investig 2019;16(1):93-96

Key Words Emotional stability, Insomnia, Anxiety, Cataract.

\section{INTRODUCTION}

Cataracts are a major cause of visual impairment with an increasing global prevalence due to an aging society. ${ }^{1,2}$ Decreases in visual acuity and vision loss in patients with cataracts lower the social and occupational functioning of individuals. Cataracts also cause insomnia-related symptoms, such shortened sleep duration and reduced sleep quality, ${ }^{3-6}$ which may lead to a deterioration in the patients' quality of life. ${ }^{7-9}$

Patients with cataracts often experience anxiety and depression associated with visual recovery before surgery, and these psychiatric symptoms could affect sleep. ${ }^{10,11}$ Patients with cataracts experience diverse psychiatric symptoms according to individual factors. ${ }^{5,11,12}$ Individual personality traits are risk factors for or protective factors against psychiatric symp-

Received: August 6, 2018 Revised: September 19, 2018

Accepted: November 13, 2018

$\bowtie$ Correspondence: Dongyun Lee, MD

Department of Psychiatry, Gyeongsang National University Changwon Hospital, 11 Samjeongja-ro, Seongsan-gu, Changwon 51472, Republic of Korea

Tel: +82-55-214-3801, Fax: +82-55-214-3254, E-mail: yuny0829@gmail.com

(c) This is an Open Access article distributed under the terms of the Creative Commons Attribution Non-Commercial License (https://creativecommons.org/licenses/bync/4.0) which permits unrestricted non-commercial use, distribution, and reproduction in any medium, provided the original work is properly cited. toms, such as depression and anxiety.

Among the various models used to evaluate personality traits, the Big Five Model, consisting of emotional stability (ES), extraversion, agreeableness, conscientiousness, and openness to experience, is widely used, ${ }^{13}$ and various studies have been conducted to investigate the relationships among medical intervention methods and these personality domains. ${ }^{14}$ Of these personality domains, low levels of ES may increase vulnerability to stress, ${ }^{15}$ and ES is significantly associated with preoperative anxiety and surgical outcomes. ${ }^{16,17}$ Therefore, the ES level of patients with cataracts may be related to anxiety and insomnia.

Previous studies have mainly focused only on evaluating depression and anxiety in cataract patients, and studies examining the relationship between personality traits, such as ES, and anxiety and insomnia are lacking. The purpose of this study was to evaluate the effect of ES on preoperative anxiety and insomnia in patients who were scheduled to undergo cataract surgery.

\section{METHODS}

The subjects were scheduled to undergo an operation for 
cataracts. Self-questionnaires evaluating sociodemographic characteristics, the level of insomnia, depressive and anxious mood, and the ES personality trait were administered to all subjects before the cataract surgery. The total number of subjects was 54 . The completed questionnaires were collected by the investigators before the surgery. This study was approved by the Institutional Review Board (approval No. 2015-01-011003). Informed consent was provided by all subjects when they enrolled.

The subjects' level of insomnia was evaluated using the Insomnia Severity Index (ISI). The ISI consists of 7 items, including satisfaction with sleep, functional impairment in daily life due to insomnia, and recognition of problematic sleep. Higher total scores represent more severe insomnia. ${ }^{18}$ The validity of the Korean version of the ISI was confirmed (Cronbach's $\alpha=0.86){ }^{19}$

ES was investigated using the International Personality Item Pool (IPIP). The IPIP was developed to evaluate individuals' personality traits, and 10 questions on the IPIP assess ES. ${ }^{13} \mathrm{~A}$ study using the Korean version of the IPIP showed that this measure has sufficient validity (Cronbach's $\alpha=0.86$ ). ${ }^{20}$

The Hospital Anxiety and Depression scale (HADS) was used to assess anxious and depressive mood symptoms. The HADS was developed to assess subjects visiting a hospital. The measure includes two subdomains, i.e., anxiety (HADS-A) and depression (HADS-D), and each subdomain consists of 7 items; in total, the HADS consists of 14 questions. Higher total scores on each subdomain indicate more severe symptoms of anxiety or depression..$^{21}$ The internal validity of the Korean version of the HADS was confirmed, with Cronbach's $\alpha=0.89 .^{22}$

The subjects were divided into the following three groups according to their ES scores using a method suggested in a previous study involving a Korean population: low ES (1 SD below the mean), average ES (within $\pm 1 \mathrm{SD}$ ), and high ES (1 SD above the mean). ${ }^{20}$ The Kruskal-Wallis test was performed to compare the scores on the ISI, HADS-A, and HADS-D among the three groups, and a Games-Howell post hoc analysis was performed to analyze the variables that did not show homogeneity. The Pearson analysis was performed to analyze the correlation of ES with the HADS-D score and the HADS total score. In addition, Spearman correlation analysis was performed to analyze the correlation of ES with the scores on the HADS-A and the ISI, the distributions for which did not show normality. A multiple linear regression analysis was performed to analyze the association between the ISI total score as the dependent variable and the ES score, the HADS-A score, and the HADS-D score as the independent variables, after adjusting for age, sex, and caffeine consumption as covariates. A pathway analysis was performed to analyze the indirect and mediating effects of the ES, HADS-A, and ISI scores. The model fit was evaluated by calculating the SRMR, NFI, IFI, and CFI. All statistical analyses were conducted using SPSS version 21.0 (IBM Corp., Armonk, NY, USA) and AMOS 18.0 (SPSS Inc., Chicago, IL, USA), and two-tailed $p$-values $<0.05$ were considered to indicate statistical significance.

\section{RESULTS}

The mean age of the subjects was $63.6 \pm 10.7($ mean \pm SD) years, and males comprised $55.6 \%$ of the sample. The mean scores of the subjects on the ISI, ES, HADS-D, and HADS-A were as follows: 7.6 $\pm 7.6,29.0 \pm 6.0,5.5 \pm 4.9$, and 7.8 \pm 4.4 . After dividing the subjects into three groups according to their ES score, the degree of insomnia was compared among the groups, and the low-ES group showed significantly higher insomnia severity than the high-ES group $(12.7 \pm 10.2$ vs. $5.1 \pm 5.7, \mathrm{p}=$ 0.048). The means of the HADS total and subscale scores significantly differed among the ES groups (Table 1). In the results of the correlation analyses, the ES score was significantly correlated with the HADS total score, the HADS-A score, the HADS-D score, and the ISI score, the r-values ( $\mathrm{p}$-value) for which were $-0.810(p<0.001),-0.693(p<0.001),-0.739(p<0.001)$, and $-0.326(\mathrm{p}=0.016)$, respectively. According to the multiple linear regression analysis, the standardized regression coefficient $(\beta)$ between the ES and ISI scores was $-0.099(p=0.609)$, and the $\beta$ between the ES and HADS-A scores was 0.697 ( $\mathrm{p}=$ 0.001 ). No statistically significant association was observed between the HADS-D and ISI scores $(\beta=-0.149, \mathrm{p}=0.421)$. The effect size (Cohen's $\mathrm{f}^{2}$ ) for the multiple linear regression analysis was 0.828 . According to the pathway analysis (Figure 1),

Table 1. Symptoms of insomnia, anxiety and depression according to the level of emotional stability

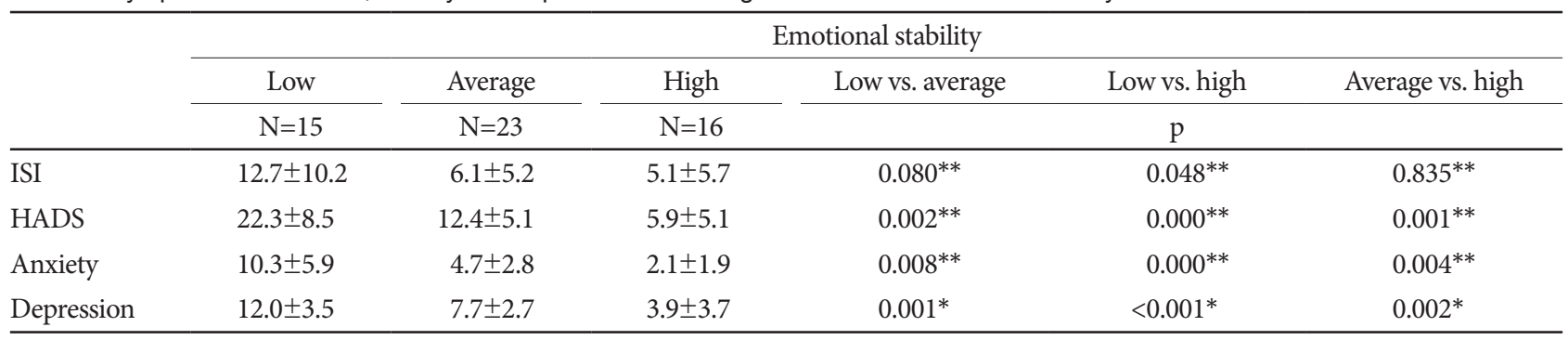

${ }^{*}$ Bonferroni post-hoc analysis, ${ }^{* *}$ Games-Howell post-hoc analysis. ISI: Insomnia Severity Index, HADS: Hospital Anxiety-Depression Scale 


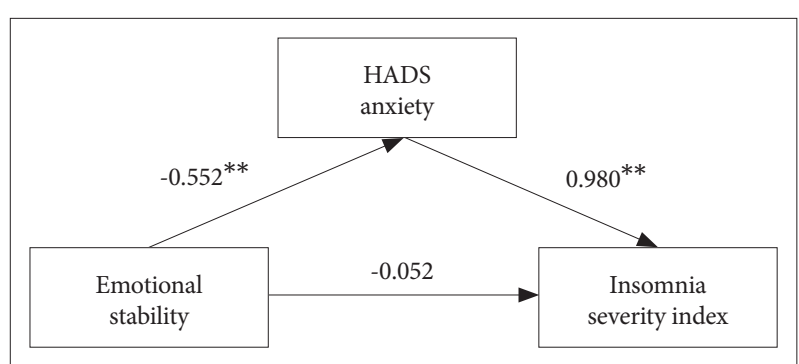

Figure 1. Pathway Analysis of the Effects of Emotional Stability on Insomnia through Anxiety Symptoms. The indirect effect of emotional stability on insomnia through anxiety symptoms was $-0.541(p=0.009)$, indicating that the mediating effect was significant. ${ }^{* *} p<0.01$. Model fit: SRMR $=0.000, N F I=1.000, I F I=1.000$, $\mathrm{CFI}=1.000 ; 200$ bootstraps; 95\% confidence intervals. HADS: Hospital Anxiety-Depression Scale, ISI: Insomnia Severity Index.

ES had a statistically significant indirect effect $(-0.541)$ on the ISI scores through the HADS-A scores ( $\mathrm{p}=0.009$, bootstrap 200; 95\% confidence intervals), and the model fit was appropriate $(\mathrm{SRMR}=0.000, \mathrm{NFI}=1.000, \mathrm{IFI}=1.000, \mathrm{CFI}=1.000)$.

\section{DISCUSSION}

In this study, we found that lower levels of ES could contribute to more severe insomnia symptoms in cataract patients before surgery. Furthermore, we revealed that anxiety had a mediating effect on the negative relationship between ES and insomnia. In a community-based study of elderly cataract patients, visual impairment was closely related to insomnia, and more than half of the patients reported sleep induction impairment and sleep maintenance difficulties. ${ }^{6}$ Patients with cataracts easily experience anxiety and depression due to the discomfort of daily life caused by a deterioration in visual function before surgery and the negative expectations of poor postoperative outcomes. ${ }^{10,13}$ Additionally, preoperative anxiety symptoms may trigger the activation of the sympathetic nervous system ${ }^{23}$ and result in an abnormal arousal state, which can adversely influence the maintenance of sleep in patients. ${ }^{24}$ Compared with emotionally disturbed individuals, people with high levels of ES are not only more tolerant of uncertain situations but are also more likely to maintain stability and practice self-control in stressful situations. ${ }^{13,20}$ The mediating effect of anxiety symptoms on the negative relationship between ES and insomnia, which is the main outcome of this study, could be explained by the protective role played by high levels of ES in preoperative anxiety.

From a neurobiological perspective, high levels of ES are positively related to the circadian rhythm, which is responsible for favorable night sleep quality. ${ }^{25}$ Patients with cataracts have decreased light input through the eyes due to visual impairment. This decreased light input has a negative effect on the suprachiasmatic nucleus (SCN) in the hypothalamus, which regulates the sleep cycle, thus causing insomnia. ${ }^{6} \mathrm{How}-$ ever, individuals with high ES maintain better serotonergic function than those with low ES. The maintenance of these serotonergic functions could stabilize the change in the circadian rhythm due to the impairment in SCN function by the light input reduction and may be protective against insomnia in cataract patients. ${ }^{25,26}$ This hypothesis may be a neurobiological explanation of the negative association between ES and insomnia in the present study.

This study has several limitations. First, assessing objective insomnia-related symptoms was challenging due to the use of self-report questionnaires. Therefore, evaluating the patients' sleep state more precisely using laboratory tools, such as polysomnography or actigraphy, is necessary. Second, because the total number of participants in this study was relatively small, the generalizability of the results to the entire population is limited. However, since the effect size (0.828) of the outcomes was large, the significance of the results appears to be sufficient.

Despite these limitations, this study is the first to reveal that ES, which is a personality trait, may be associated with preoperative insomnia in cataract patients. Previous studies investigating psychiatric symptoms in cataract patients have been limited to anxiety and depression and did not evaluate personality traits. ${ }^{5,11,12}$ Anxiety symptoms in patients with cataracts decrease the psychological and physical quality of life8 and adversely affect the course and prognosis of the disease. ${ }^{27}$ Screening for factors associated with anxiety, such as ES, in cataract patients could be important. In this study, we revealed a negative relationship between ES and preoperative anxiety. Thus, if the ES level of the patient is assessed before surgery and psychological stability is facilitated using appropriate interventions for patients with low ES, then surgical outcomes and quality of life are expected to improve.

\section{REFERENCES}

1. Ramke J, Gilbert CE, Lee AC, Ackland P, Limburg H, Foster A. Effective cataract surgical coverage: an indicator for measuring quality-ofcare in the context of Universal Health Coverage. PLoS One 2017; 12:e0172342.

2. World Health Organization. Blindness and visual impairment. Available at: http://www.who.int/en/news-room/fact-sheets/detail/blindness-and-visual-impairment. Accessed October 13, 2017.

3. Peltzer K, Phaswana-Mafuya N. Association between visual impairment and low vision and sleep duration and quality among older adults in South Africa. Int J Environ Res Public Health 2017;14. pii: E811.

4. Wang JJ, Mitchell P, Smith W, Cumming RG, Attebo K. Impact of visual impairment on use of community support services by elderly persons: the Blue Mountains Eye Study. Invest Ophthalmol Vis Sci 1999; 40:12-19.

5. An Y, Joo CK. The U-shaped association between self-reported sleep duration and visual impairment in Korean adults: a population-based study. Sleep Med 2016;26:30-36.

6. Zizi F, Jean-Louis G, Magai C, Greenidge KC, Wolintz AH, Heath-Phil- 
lip O. Sleep complaints and visual impairment among older Americans: a community-based study. J Gerontol A Biol Sci Med Sci 2002;57:M691M694.

7. Berle D, Steel Z, Essue BM, Keay L, Jan S, Tan Phuc HT, et al. Multisite prospective investigation of psychological outcomes following cataract surgery in Vietnam. BMJ Glob Health 2017;2:e000162.

8. Fazzi L, Dobrianskyj FM, Reggi JR, Otani VH, Otani TZ, Uchida RR. The influence of anxiety on quality of life among patients with an indication for cataract surgery. Sao Paulo Med J 2015;133:160-161.

9. Skiadaresi E, McAlinden C, Pesudovs K, Polizzi S, Khadka J, Ravalico G. Subjective quality of vision before and after cataract surgery. Arch Ophthalmol 2012;130:1377-1382.

10. Freeman EE, Gresset J, Djafari F, Aubin MJ, Couture S, Bruen R, et al. Cataract-related vision loss and depression in a cohort of patients awaiting cataract surgery. Can J Ophthalmol 2009;44:171-176.

11. Seixas A, Ramos AR, Gordon-Strachan GM, Fonseca VA, Zizi F, JeanLouis G. Relationship between visual impairment, insomnia, anxiety/ depressive symptoms among Russian immigrants. J Sleep Med Disord 2014;1. pii: 1009.

12. Lundström M, Pesudovs K. Questionnaires for measuring cataract surgery outcomes. J Cataract Refract Surg 2011;37:945-959.

13. Goldberg LR. A broad-bandwidth, public domain, personality inventory measuring the lower-level facets of several five-factor models. Pers Psychol Eur 1999;7:7-28.

14. Borges NJ, Savickas ML. Personality and medical specialty choice: a literature review and integration. J Career Assess 2002;10:362-380.

15. Jylhä P, Isometsä E. The relationship of neuroticism and extraversion to symptoms of anxiety and depression in the general population. Depress Anxiety 2006;23:281-289.

16. Jess P, Jess T, Beck H, Bech P. Neuroticism in relation to recovery and persisting pain after laparoscopic cholecystectomy. Scand J Gastroen- terol 1998;33:550-553.

17. Thorp JM, Kennedy BW, Millar K, Fitch W. Personality traits as predictors of anxiety prior to caesarean section under regional anaesthesia. Anaesthesia 1993;48:946-950.

18. Morin CM. Insomnia: Psychological Assessment and Management. New York, NY: Guilford Press; 1994.

19. Cho YW, Song ML, Morin CM. Validation of a Korean version of the insomnia severity index. J Clin Neurol 2014;10:210-215.

20. Garrison YL, Lee KH. Meaning in life among Korean college students based on emotionality and tolerance of uncertainty. Pers Individ Dif 2017;112:26-30.

21. Zigmond AS, Snaith RP. The hospital anxiety and depression scale. Acta Psychiatr Scand 1983;67:361-370.

22. Oh SM, Min KJ, Park DB. A study on the standardization of the hospital anxiety and depression scale for Koreans: a comparison of normal, depressed and anxious groups. J Korean Neuropsychiatr Assoc 1999; 38:289-296

23. Bonnet MH, Arand DL. Hyperarousal and insomnia: state of the science. Sleep Med Rev 2010;14:9-15.

24. Saper CB, Cano G, Scammell TE. Homeostatic, circadian, and emotional regulation of sleep. J Comp Neurol 2005;493:92-98.

25. DeYoung CG, Hasher L, Djikic M, Criger B, Peterson JB. Morning people are stable people: circadian rhythm and the higher-order factors of the Big Five. Pers Individ Dif 2007;43:267-276.

26. Yuan Q, Lin F, Zheng X, Sehgal A. Serotonin modulates circadian entrainment in Drosophila. Neuron 2005;47:115-127.

27. El-Gabalawy R, Mackenzie CS, Shooshtari S, Sareen J. Comorbid physical health conditions and anxiety disorders: a population-based exploration of prevalence and health outcomes among older adults. Gen Hosp Psychiatry 2011;33:556-564. 